DRAFT VERSION JANUARY 28, 2002

Preprint typeset using LATEX style emulateapj v. 16/07/00

\title{
THE VELOCITY DISTRIBUTION OF ISOLATED RADIO PULSARS
}

\author{
Z. ARzoumanian ${ }^{1}$ \\ Laboratory for High-Energy Astrophysics, NASA-GSFC, Greenbelt, MD 20771 \\ D. F. CHERNOFF \\ Center for Radiophysics \& Space Research, Cornell University, Ithaca, NY 14853 \\ J. M. CORDES \\ Astronomy Department and NAIC, Comell University, Ithaca, NY 14853
}

Draft version January 28, 2002

\begin{abstract}
We infer the velocity distribution of radio pulsars based on large-scale $0.4 \mathrm{GHz}$ pulsar surveys. We do so by modelling evolution of the locations, velocities, spins, and radio luminosities of pulsars; calculating pulsed flux according to a beaming model and random orientation angles of spin and beam; applying selection effects of pulsar surveys; and comparing model distributions of measurable pulsar properties with survey data using a likelihood function. The surveys analyzed have well-defined characteristics and cover $\sim 95 \%$ of the sky. We maximize the likelihood in a 6-dimensional space of observables $P, \dot{P}, \mathrm{DM},|b|, \mu, F$ (period, period derivative, dispersion measure, Galactic latitude, proper motion, and flux density). The models we test are described by 12 parameters that characterize a population's birth rate, luminosity, shutoff of radio emission, birth locations, and birth velocities. We infer that the radio beam luminosity $(i)$ is comparable to the energy flux of relativistic particles in models for spin-driven magnetospheres, signifying that radio emission losses reach nearly $100 \%$ for the oldest pulsars; and (ii) scales approximately as $\dot{E}^{1 / 2}$ which, in magnetosphere models, is proportional to the voltage drop available for acceleration of particles. We find that a two-component velocity distribution with characteristic velocities of $90 \mathrm{~km} \mathrm{~s}^{-1}$ and $500 \mathrm{~km} \mathrm{~s}^{-1}$ is greatly preferred to any one-component distribution; this preference is largely immune to variations in other population parameters, such as the luminosity or distance scale, or the assumed spin-down law. We explore some consequences of the preferred birth velocity distribution: $(i)$ roughly $50 \%$ of pulsars in the solar neighborhood will escape the Galaxy, while $\sim 15 \%$ have velocities greater than 1000 $\mathrm{km} \mathrm{s}^{-1}$; (ii) observational bias against high velocity pulsars is relatively unimportant for surveys that reach high Galactic $|z|$ distances, but is severe for spatially bounded surveys; (iii) an important low-velocity population exists that increases the fraction of neutron stars retained by globular clusters and is consistent with the number of old objects that accrete from the interstellar medium; (iv) under standard assumptions for supernova remnant expansion and pulsar spin-down, $\sim 10 \%$ of pulsars younger than $20 \mathrm{kyr}$ will appear to lie outside of their host remnants. Finally, we comment on the ramifications of our birth velocity distribution for binary survival and the population of inspiraling binary neutron stars relevant to some GRB models and potential sources for LIGO.
\end{abstract}

Subject headings: pulsars:general-methods:statistical

\section{INTRODUCTION}

Only a small fraction of the estimated $10^{9}$ neutron stars (NS) in the Galaxy are visible to Earth-bound observers as radio pulsars. In addition to source brightness and survey sky coverage, many effects unique to neutron stars determine which objects will be detected: (1) highly beamed emission; (2) turning-off of radio emission as a NS spins down; (3) migration from a thin extreme Population I $z$ distribution at birth to a combined thick disk and unbounded population; (4) interstellar plasma dispersion and scattering that distort pulses, diminishing search sensitivities to distant and rapidly rotating pulsars. Statistical studies that aim to determine the birthrate, total number, and spatial distribution of radio pulsars must contend with these selection effects. Indeed, previous studies (some recent examples are Lyne et al. 1998; Hartman et al. 1997; Hansen \& Phinney 1997; Lorimer, Bailes \& Harrison 1997) differ from one another significantly in this respect. All analyses have been ham- pered by our incomplete knowledge of intrinsic properties such as the luminosities and shapes of pulsar beams and their variations with spin rate and magnetic field strength.

We report a new effort to derive the maximum useful information about Galactic neutron stars from the subset observable as radio pulsars (excluding millisecond and binary pulsars and those in globular clusters), simultaneously solving for many of the pulsar properties upon which the effects of survey selection depend. We use a new, highly realistic model of NS birth, evolution, and detection in radio surveys, and we implement a rigorous statistical analysis of the joint distribution of observable pulsar properties drawn from real surveys. Monte Carlo simulations of NS populations are tested for detectability and compared, in a 6-dimensional space of observables, to the observed pulsar sample. We vary the model parameters to infer most likely values and credible ranges. To the extent that realworld selection effects are adequately described in the model, 
the analysis yields results that are independent of selection biases.

The main focus of this paper is a determination of the pulsar velocity distribution function at birth and its implications. Other results, such as the luminosities of pulsar beams and the radio pulsar birthrate, are briefly described here (covariances do exist between these model parameters and the best-fit velocity results), but a detailed exposition of the model, statistical method, and insights into other areas of pulsar phenomenology will be presented elsewhere. We summarize the essential features of our model and method in Sec. 2, present our results in Sec. 3, and discuss some implications of our new determination of the pulsar birth velocity distribution in Sec. 4 .

\section{MODEL AND METHOD}

\subsection{Summary of the Model}

We model the birth properties and rotational, kinematic, and luminosity evolution of a Monte Carlo population of neutron stars. The characteristics of eight real-world surveys are then used to assess the detectability of each simulated NS as a radio pulsar. The model-derived population is compared to the actual survey results using a likelihood analysis.

\subsubsection{Neutron Star Birth}

We assume that the NS birthrate is constant in time, uniform per unit area for a Galactic disk with $15 \mathrm{kpc}$ radius, and exponentially distributed above and below the disk midplane with parameterized scale $z_{0}$. The orientations of stellar spin and magnetic axes are independent and uniformly distributed over the sphere. The birth velocity is distributed isotropically in direction about the local standard of rest; it incorporates both an asymmetric "kick" impulse from a supernova explosion and the residual contribution from a disrupted binary, for those objects born in binaries. We do not separately investigate the two phenomena here nor do we attempt to model neutron stars that remain bound in binaries (high-field pulsars in binaries are a negligible population). Two model distributions of the velocity magnitude are considered, consisting of one or two Gaussian components. The two component model is

$$
\begin{aligned}
f(v) & =4 \pi v^{2}\left[w_{1} \frac{1}{\left(2 \pi \sigma_{v_{1}}^{2}\right)^{3 / 2}} \exp \left(-v^{2} / 2 \sigma_{v_{1}}^{2}\right)\right. \\
& \left.+\left(1-w_{1}\right) \frac{1}{\left(2 \pi \sigma_{v_{2}}^{2}\right)^{3 / 2}} \exp \left(-v^{2} / 2 \sigma_{v_{2}}^{2}\right)\right],
\end{aligned}
$$

parameterized by $\sigma_{v_{1}}$ and $\sigma_{v_{2}}$, the dispersions of the low- and high-velocity components, respectively, and by $w_{1}$, the fraction of neutron stars with parent Gaussian of width $\sigma_{v_{1}}$. Finally, the initial periods and magnetic field strengths are drawn from lognormal distributions with parameterized means $\left(\left\langle\log P_{0}\right\rangle\right.$, for $P_{0}$ in seconds, and $\left\langle\log B_{0}\right\rangle$, for $B_{0}$ in Gauss), widths $\left(\sigma_{\log P_{0}}\right.$, $\left.\sigma_{\log B_{0}}\right)$, and fixed high and low cutoffs.

\subsubsection{Evolution.}

Given initial conditions, the evolution of a new-born pulsar to the present epoch is deterministic and described as follows. Most of our analysis assumes spin down according to a constant, magnetic-dipole braking torque ${ }^{2}$ with "braking index" $n=3$. This choice facilitates comparison of our results with previous NS population studies, but we have also investigated an alternate torque model, with $n=4.5$-effects of different assumptions for $n$ are described in Sec. 3.4. We assume that the direction of the magnetic field with respect to the spin axis does not evolve with time. Each star moves in the Galactic potential given by Paczyǹski (1990) for the disk and bulge, and by Caldwell \& Ostriker (1981) for the corona.

Each star's luminosity is a function of period and spin-down rate, with no intrinsic spread (i.e., pulsars are assumed to be standard candles for fixed values of $P$ and $\dot{P}$ ),

$$
L_{r}=\min \left\{P^{\alpha} \dot{P}_{15}^{\beta} L_{0}, \dot{E}\right\} \operatorname{erg~s}^{-1},
$$

where $\left(\alpha, \beta, L_{0}\right)$ are model parameters, $\dot{P}=\dot{P}_{15} 10^{-15} \mathrm{~s} \mathrm{~s}^{-1}$, and $\dot{E}=4 \pi^{2} I P / P^{3}$ is the spin-down energy loss rate for moment of inertia $I=10^{45} \mathrm{~g} \mathrm{~cm}^{2}$. Previous analyses have assumed intrinsic spreads in the "pseudo-luminosity" (flux $\times$ distance-squared) to account for viewing of the pulsar beam from different orientations as well as possible differences in intrinsic luminosity. We choose instead to explicitly model flux variations due to viewing geometry through a beam model, described below, and a physically meaningful luminosity (in $\mathrm{erg} \mathrm{s}^{-1}$ ). We find that the inevitable spread in fluxes due to viewing geometry is substantial. Some implications of this choice are discussed in Sec. 3.4.

Slowly rotating neutron stars do not emit in the radio because the electric field near the polar cap is too small (e.g., Chen \& Ruderman 1993). The cutoff may not be abrupt; we implement a "death band" as the probability that objects with given $\dot{P} / P^{3}$ are radio quiet. The probability is

$$
1 / 2\left\{\tanh \left[\left(\dot{P_{15}} / P^{3}-10^{\overline{\mathrm{DL}}}\right) / \sigma_{\mathrm{DL}}\right]+1\right\}
$$

for $P$ in seconds, so that the position and lateral extent of the death band are parameterized by $\overline{\mathrm{DL}}$ and $\sigma_{\mathrm{DL}}$.

\subsubsection{Beaming and Pulse profiles.}

For each simulated neutron star, a pulse profile is generated using a phenomenological radio-beam model. Our beam model was fixed by fitting $0.4 \mathrm{GHz}$ pulse profiles to ten known pulsars with viewing geometries that are well-determined from polarization measurements (e.g., Lyne \& Manchester 1988; Rankin 1993), and which span much of the $P-\dot{P}$ region of interest: PSRs $\mathrm{B} 0301+19, \mathrm{~B} 0329+54, \mathrm{~B} 0450-18, \mathrm{~B} 0525+21, \mathrm{~B} 1541+09$, B 1642-03, B1821+05, B 1933+16, B2002+31, B2111+46. Satisfactory fits were achieved with the following beam description, which (together with our view that pulsars are standard candles for given $P, \dot{P}$ ) is consistent with preliminary results of a more ambitious analysis of pulse waveforms and luminosities to be presented elsewhere.

In our model, pulsed radio flux from a NS depends on contributions from one "core" and one "conal" beam. Each is Gaussian in form with opening angle $\rho$ (half-width at $1 / e$ ) that scales with period as

$$
\begin{aligned}
\rho_{\text {core }} & =1.5^{\circ} P^{-0.5} \\
\rho_{\text {cone }} & =6.0^{\circ} P^{-0.5} .
\end{aligned}
$$

The beams subtend solid angles $\Omega=f \pi \rho^{2} / \ln 2$, where the correction factor $f_{\text {cone }}=0.4$ accounts for the hollowness of the cone beam, and $f_{\text {core }}=1$. The total radio luminosity $L_{r}$ is apportioned such that the ratio of core to cone peak flux is

$$
F_{\text {core }} / F_{\text {cone }}=\frac{20}{3} P^{-1} \text {. }
$$

We omit from our spin-down expression the $\sin ^{2} \alpha$ dependence of the idealized magnetic dipole braking torque, where $\alpha$ is the angle between the spin and magnetuc field axcs. In magnetosphere models (e.g. Goldreich \& Julian 1969), the torque does not vanish for aligned rotators $(\alpha=0)$. 
The core increases in strength with decreasing period and decreasing observing frequency $\nu$; the latter dependence is not relevant to the present analysis of $0.4 \mathrm{GHz}$ surveys. An example of the distribution of core and conal flux is shown in Fig. 1.

Pulse waveforms are constructed for the viewing geometry of each Monte Carlo pulsar, with two oppositely-directed radio beams centered on the star's magnetic axis. The waveform flux at pulse phase $\phi$ corresponding to polar angle $\theta(\phi)$ to the line of sight is

$$
F(\phi)=F_{\text {core }} \exp \left\{-\theta^{2} / \rho_{\text {core }}^{2}\right\}+F_{\text {cone }} \exp \left\{-(\theta-\bar{\theta})^{2} / w_{e}^{2}\right\},
$$

where $\vec{\theta}$ and $w_{e}$ are the centroid and effective width of the Gaussian conal beam. Figure 2 shows typical differential and cumulative distributions of period-averaged flux density that result from our beam model and radio luminosity inferred from the likelihood analysis (Sec. 3). The highest fluxes correspond to nearly aligned viewing geometries, while the tail at low flux corresponds to lines of sight grazing the skirts of the radio beam. The two peaks in the differential distribution arise from the core (narrower beam, higher average flux) and cone (wider beam, lower average flux) components.

\subsubsection{Detection.}

The effects of interstellar dispersion and scattering are applied to the pulse profile of each potentially detectable NS, where the degree of waveform "smearing" depends on the applicable survey instrumentation. The DM and SM values used are based on the Taylor \& Cordes (1993) model, dithered to simulate errors in the DM-distance relationship at the $50 \%$ level. Pulse profiles are Fourier transformed and tested for detection by each survey with sky coverage in the direction of the simulated pulsar. The minimum detectable flux is computed for the antenna gain, receiver and sky temperature, bandwidth, dwell time, sample interval, and detection threshold (including harmonic summing) applicable to each survey. Detection probabilities were modified by the ratio of successfully searched area to the area contained within the survey boundaries on the sky. We do not model specific telescope pointings, but assume uniform antenna gain across the region surveyed by each telescope. In reality, antenna gain drops off away from the pointing direction, and survey pointings are generically arranged in a close grid with adjacent pointings separated by the half-power width of one, roughly Gaussian, telescope "beam." The assumption of constant gain results in an enhanced detection rate and, hence, a lower inferred birthrate, which we later correct by the ratio of volumes for beams with constant and Gaussian cross-sections, $f_{\text {gain }}=1.4$.

In summary, we draw neutron stars independently from a parameterized parent population, evolve them according to the simulation model and test for detectability as pulsars in the given surveys. This "forward evolution" from birth to detection yields distributions of simulated pulsar properties that may be compared to those of the observed sample.

\subsection{Surveys and Data: Selection Criteria}

The surveys modeled, all at or near $0.4 \mathrm{GHz}$, are those of Camilo, Nice \& Taylor (1996), Dewey et al. (1985), Foster et al. (1995), Manchester et al. (1978), Manchester et al. (1996), Nice, Fruchter \& Taylor (1995), Stokes et al. (1985), and Thorsett et al. (1993). Together, they cover some $95 \%$ of the sky. The data are culled from the May 1995 electronic version of the Taylor et al. $(1993 ; 1995)$ catalog of 721 pulsars, supplemented with $66 \dot{P}$ measurements from D'Amico et al. (1998), including the $8.5 \mathrm{sec}$ pulsar of Young et al. (1999). Of the full set of known pulsars we keep only those that were detected in at least one of these surveys; for example, we exclude objects found in deep searches that targeted supernova remnants or X-ray sources, and pulsars detected only in high-frequency (1.4 GHz or higher) searches. In addition, to avoid contamination from objects with complicated evolutionary histories, we exclude from our analysis: binary pulsars, pulsars in globular clusters, extragalactic (LMC and SMC) pulsars, and all pulsars with spin-down rates less than $10^{-16} \mathrm{~s} \mathrm{~s}^{-1}$ because of potential confusion with spun-up objects. The resulting dataset contains $N_{\text {psr }}=435$ pulsars, 79 of which have measured proper motions or useful upper bounds. An additional 54 cataloged pulsars meet the criteria above but do not have measured spin-down rates. We account for these objects in our derived birthrate through a correction factor $f_{\text {no } \dot{P}}=(435+54) / 435$, but otherwise neglect them in the likelihood analysis.

\subsection{Likelihood Function}

The distributions of measured quantities that characterize pulsars arise from variations intrinsic to the NS population, bias in survey selection, and measurement errors. We account for measurement errors as reported in the literature, and model intrinsic variations through Monte Carlo (MC) realizations subject to the same detection criteria used to obtain the observed pulsar sample. Measurable quantities ("observables") are of two types: (a) $\mathcal{D}_{a}=\{P, \dot{P},|b|, \mathrm{DM}\}$, which are known with sufficient precision that their distributions are determined by the biased sampling of the population; and (b) $\mathcal{D}_{b}=\{\mu, F\}$, proper motion and flux density, which carry significant measurement uncertainty described by observer-reported or assumed likelihood functions $\mathcal{L}_{\{\mu, F\}}^{\text {obs }} \equiv \mathcal{L}_{\mu}^{\text {obs }} \mathcal{L}_{F}^{\text {obs }}$. We assume a Gaussian form for $\mathcal{L}_{\mu}^{\text {obs }}$, and log-normal distributions above a detection threshold for $\mathcal{L}_{F}^{\text {obs }}$.

We do not know a priori the form of the likelihood function for $\mathcal{D}_{a}$, and therefore construct it numerically. We first partition the 4-dimensional space for $\mathcal{D}_{a}$ using the real sample of pulsars to define bins in a heirarchical fashion-each bin corresponds to a small volume element centered on specific values of $P, \dot{P},|b|$, and DM. We then create MC pulsars according to a specific population/evolution/luminosity model, and find the total weight $W_{i}$ of detected objects with observables that fall within the boundaries of the $i$-th bin. For birthrate $\dot{N}$, the expected number of such objects is $\left\langle n_{i}\right\rangle=\dot{N}\left(T_{\mathrm{MC}} / W\right) W_{i}$. Here, $W$ is the total weight of MC neutron stars born uniformly distributed over time interval $T_{\text {MC }}$. The likelihood for the $\mathcal{D}_{a}$ dataset is then formed using products of Poisson probabilities for 0 or 1 elements in each bin,

$$
\mathcal{L}_{a}=e^{-\langle n\rangle} \prod_{i}\left\langle n_{i}\right\rangle,
$$

where the product is restricted to occupied bins, and the total expected number of pulsars is

$$
\langle n\rangle=\sum_{i}\left\langle n_{i}\right\rangle .
$$

For precisely measured quantities, the numerical evaluation of $\mathcal{L}_{a}$ is exact in the limit of zero bin size and small occupancy. In practice, we use bins that enclose more than one data pulsar, and we generalize the likelihood function accordingly 
Information from $\mathcal{D}_{b}$ observables must also be included. The weight of Monte Carlo points in the $i$-th bin is $W_{i}=$ $\sum_{k} w_{k}$ (where $k$ counts individual $\mathrm{MC}$ points); the weight of points consistent with the observer-reported uncertainties is $\sum_{k} w_{k} \mathcal{L}_{\{\mu, F\}_{k}}^{\text {obs }}$ (sum over joint likelihood of proper motion and flux for data in the $i$-th bin), which may be rewritten as $W_{i}\left(\sum_{k} w_{k} \mathcal{L}_{\{\mu, F\}_{k}}^{\text {obs }} / \sum_{k} w_{k}\right)=W_{i}\left\langle\left\langle\mathcal{L}_{i}^{\text {obs }}\right\rangle\right\rangle$. The expected number of pulsars in the $i$-th bin consistent with the flux and proper motion observations is $N\left(T_{\mathrm{MC}} / W\right) W_{i}\left\langle\left\langle\mathcal{L}_{i}^{\text {obs }}\right\rangle\right\rangle$. The likelihood of the whole dataset of precisely and imprecisely measured quantities is then $\mathcal{L}=e^{-\langle n\rangle} \prod_{i}\left\langle n_{i}\right\rangle\left\langle\left\langle\mathcal{L}_{i}^{\text {obs }}\right\rangle\right\rangle$.

Maximizing $\Lambda=\ln \mathcal{L}$ with respect to $\dot{N}$ yields the birthrate

$$
\dot{N}_{\mathrm{ML}}=\left(\frac{N_{d}}{W_{d}}\right)\left(\frac{W}{T_{\mathrm{MC}}}\right),
$$

where $N_{d}=435$ is the number of actual pulsars, $W_{d}$ is the weight of detected Monte Carlo points, $W$ is the total weight of Monte Carlo points and $T_{\mathrm{MC}}$ is the timescale of the simulation. In this paper we analyze the model parameters using the likelihood evaluated at the most likely birthrate $\dot{N}_{\mathrm{ML}}$ :

$$
\left.\Lambda\right|_{\dot{N}_{\mathrm{ML}}}=\sum_{i} \ln \left(\frac{W_{i}}{W_{d}}\right)+\sum_{i} \ln \left\langle\left\langle\mathcal{L}_{i}^{\mathrm{obs}}\right\rangle\right\rangle,
$$

where a constant term has been omitted.

Given the likelihood function (eq. 10), we employ the techniques of Bayesian inference to derive plausible ranges for model parameters, and to compare models with different numbers of parameters. (For a review of the essentials of Bayesian inference, see Gregory \& Loredo 1992.) Bayes's theorem states that the posterior probability distribution of a model $M$ given a dataset $\mathcal{D}$ is proportional to the probability of the data given the model, $P(\mathcal{D} \mid M, I)$-i.e., the likelihood $\mathcal{L}$-times the prior probability of the model $P(M \mid I)$, where $I$ represents assumptions within the model. We assume flat priors over finite ranges of the model parameters, so that the peak of the posterior is just the maximum of $\mathcal{L}$. We derive an uncertainty estimate (or "credible region," in the parlance of Gregory \& Loredo) for each model parameter from the marginal probability distribution for that parameter, i.e., from the likelihood function integrated over all other parameters. Similarly, a global likelihood for each model may be derived by integrating over all model parameters-such global likelihoods offer a rigorous means of comparison between models that have different degrees of complexity as reflected in the number of model parameters. Ratios of global likelihoods between models are known as "odds ratios."

\subsection{Implementation}

For the $\mathcal{D}_{a}$ observables, we defined a 4-dimensional space with 4 partitions in each dimension. With this choice, the 435 known pulsars were distributed into 164 distinct bins, filling $64 \%$ of the observable phase space. We found that the addition of a fifth dimension, Galactic longitude $l$, to the space of observables, or the use of two orthogonal components of proper motion ( $\mu_{l}$ and $\mu_{b}$, say) rather than the magnitude, yielded too few MC points in some bins.

We tested our method by generating simulated pulsar data "catalogs" according to a particular luminosity and velocity model and attempting to recover the model parameters. The mock datasets were constructed to resemble our true dataset, both in the number of pulsars and in the number and quality of proper-motion measurements. We found that the resulting maximum likelihood parameter values differed from their expected values by no more than $20 \%$, with the $99 \%$ "credible region" of the marginalized posterior probability distribution for each parameter (see Sec. 2.3) implying uncertainties of $10 \%$ for the luminosity parameters $\beta$ and $\log L_{0}, 15 \%$ for $\alpha$, and $30 \%$ for each of the velocity parameters $\left(\sigma_{v_{1}}, \sigma_{v_{2}}, w_{1}\right)$. The model that described the mock dataset was reliably contained, in a halfdozen runs of the simulation, within the volume of the likelihood function that contained at most $90 \%$ of the probability. Further details on mock data recovery tests will be presented elsewhere.

For the likelihood analysis, we generated a Monte Carlo dataset of nearly $10^{10}$ pulsars for $T_{\mathrm{MC}}=1 \mathrm{Gyr}$, drawing the points from a large range of parameter values; the large value of $T_{\mathrm{MC}}$ was chosen to readily accomodate the oldest (characteristic age $\sim 300 \mathrm{Myr}$ ) cataloged pulsars and a range of braking models. We tested each point for detection in any one of the eight surveys and used the resultant bin occupancies to derive the likelihood function, $\mathcal{L}$, as defined above. We tested the numerical stability of $\mathcal{L}$ for the best models by increasing the number of $\mathrm{MC}$ points.

Computations were carried out on the 136-node IBM SP2 supercomputer at the Cornell Theory Center; evaluation of a single set of model parameters typically required 2.5 minutes on 48 nodes running in parallel. Computational limitations precluded a full 12-dimensional grid search. Instead, we evaluated models on 12-dimensional "crosses," iteratively repositioning the cross in search of a global maximum. We also searched over 3-and 5-dimensional sub-grids of the full parameter space, exploring in particular the velocity $\left(\sigma_{v_{1}}, \sigma_{v_{2}}, w_{1}\right)$ and luminosity $\left(\alpha, \beta, L_{0}\right)$ parameters. We have found a well-defined global maximum in the posterior probability function for models given the data.

\section{RESULTS}

\subsection{Birth Properties}

Table 1 displays the model parameter values that maximize the posterior probability for models given the data (with flat priors) over the indicated ranges. The Galactic birthrate of NSs visible as radio pulsars resulting from eq. 9 is

$$
\dot{N}=f_{\text {gain }} \times f_{\text {no } \dot{P}} \times \dot{N}_{\mathrm{ML}}=(760 \mathrm{yr})^{-1} \text {. }
$$

The first two factors in eq. 11 represent the only parts of the analysis that are not explicitly modeled by Monte Carlo, i.e., the corrections for cataloged pulsars with unmeasured spin-down rates and for variations in antenna gain as described above. These should not be confused with the more typical beaming corrections. Because our model incorporates a specific description of the radio beam, no post facto beaming correction is needed (or allowed), as it is in previous studies. Nonetheless, the value of $\dot{N}$ under-represents the true Galactic radio pulsar birthrate. As shown in Table 1, we impose cutoffs on the birth magnetic field $\left(B_{0}\right)$ distribution corresponding to the highest and lowest field strengths in our sample of cataloged pulsars; any unseen pulsars lying outside of this range have not been accounted for by our $\dot{N}$. The value of $\dot{N}$ will also depend on details of the radial distribution of NS birthsites in the Galactic disk, which we have fixed in our analysis ( $\mathrm{Sec}, 2.1 .1$ ), on our description of the pulsar beam (e.g., the assumption of circularity; Sec. 2.1.3), and on the assumed spin-down law. We compare our birthrate with previous estimates in Sec. 4.5 . 
The distribution of spin periods at birth in our model is poorly constrained by the data. It is consistent with birth at rapid rotation rates (as dictated by the presence of the Crab and Vela pulsars in our dataset), but the assumed uni-modal (log-normal) form for the distribution cannot test the hypothesis of "injection" of pulsars born with long rotation periods (Narayan \& Ostriker 1990). The birth field-strength distribution, by contrast, is very well constrained, with the mean of the log-normal distribution roughly consistent with the (present-day) mean fieldstrength for our dataset $\left(10^{12.1} \mathrm{G}\right)$ as expected, given our assumption of no torque decay. Cut-offs in the $P_{0}$ and $B_{0}$ distributions at low and high values were fixed for all models, as shown in Table 1. Finally, the preferred birth scale-height in our model is $z_{0} \sim 160 \mathrm{pc}$, consistent with the results of Cordes \& Chernoff (1998).

\subsection{Radio Luminosity}

The luminosity law with maximum posterior probability is

$$
L_{r}=P^{-1.3} P_{15}^{0.4} 10^{29.3} \mathrm{erg} \mathrm{s}^{-1} \text {, }
$$

for emissions in the radio band $(\nu>50 \mathrm{MHz})$ with power-law flux spectra. Comparison of our result with earlier statistical analyses is made difficult by the fundamental differences between our model and previous descriptions of pulsar luminosities. Note, however, that for luminosity proportional to magnetic field strength, the exponents would take on the values $(\alpha, \beta)=(0.5,0.5)$, while scaling with the spin-down energy loss rate would imply $(\alpha, \beta)=(-3,1)$. We find instead that the luminosity scaling with spin parameters is similar to that for the vacuum potential drop across the magnetic polar cap of a neutron star (e.g., Goldreich \& Julian 1969), $L_{r} \propto \dot{E}^{1 / 2}$.

$L_{r}$ is comparable in value to particle energy loss rates $\dot{E}_{p}$ calculated in "polar cap" models for the magnetosphere. For example, the energy flux in particles derived by Ruderman \& Sutherland (1975; their Eq. 29) may be written as $\dot{E}_{p}=$ $P^{-1.71} \dot{P}_{15}^{0.43} 10^{30.0} \mathrm{erg} \mathrm{s}^{-1}$, while Arons \& Scharlemann (1979) construct a "diode" model which implies $\dot{E}_{p}=P^{-1.45} P_{15}^{0.4} 10^{31.2}$ $\mathrm{erg} \mathrm{s}^{-1}$. Comparison of $L_{r}$ with $\dot{E}_{p}$ suggests that the efficiency of radio emission alone (i.e., not including $\mathrm{X}$ - and $\gamma$-ray emission) approaches unity as pulsars age. A more model-independent statement can also be made: it is conceivable that the death band is related to an upper bound in the efficiency with which spin energy is converted to radio emission. If we write $L_{r}=\epsilon_{r} \dot{E}$ $\left(\epsilon_{r} \leq 1\right)$, our luminosity law implies an efficiency of at least $\epsilon_{r}=2 \%$ for pulsars near the point in the $P-\dot{P}$ diagram where they appear to shut off $\left(P \sim 1 \mathrm{~s}, \dot{P}_{15} \sim 0.1\right)$; the largest implied efficiency among the pulsars in our dataset is $\epsilon_{r}=30 \%$ for J2144-3933, the most slowly-rotating radio pulsar known ( $P=8.5 \mathrm{~s}$; Young et al. 1999). The requirement that $L_{r} \leq \dot{E}$ implies a lower bound $\dot{P}_{15} \geq 0.10 \epsilon_{0.02}^{-1.7} P^{2.8}$, a scaling with period not unlike the death band we have assumed in our model.

We have chosen a death-band slope of $3\left(\log \dot{P}_{15}=3 \log P+\right.$ $\overline{\mathrm{DL}}$ describes the center of the death-band) in order to constrain the efficiency of radio luminosity. Theoretical expectations for the slope, however, generally lie between 2 and 3 (e.g., Zhang, Harding \& Muslimov 2000). To investigate possible dependence of the form of $L_{r}$ on the assumed death-band slope, we have evaluated luminosity models (maximizing the likelihood over a grid in $\left(\alpha, \beta, L_{0}\right)$ with all other parameters fixed) for a handful of death-band slopes in this range-we find that the maximum-likelihood values of the luminosity parameters remain essentially unchanged. We will parameterize the death- band slope in future work. For the assumed $\dot{P} / P^{3}$ line, the maximum likelihood model implies $\overline{\mathrm{DL}}=0.5$, and $\sigma_{\mathrm{DL}}=1.4$.

Finally, we note that the high-energy ( $h \nu \gtrsim 1 \mathrm{eV})$ luminosities of seven pulsars detected with the EGRET $\gamma$-ray telescope also seem to scale as $\dot{E}^{1 / 2}$ (Thompson et al. 1999 and references therein).

\subsection{Birth Velocities}

The maximum likelihood one- and two-component birth velocity distributions are displayed in Fig. 3, together with contours of log-likelihood evaluated in the vicinity of the peak. For the two-component model, $\sim 50 \%$ of pulsars have 3dimensional velocities greater than $500 \mathrm{~km} \mathrm{~s}^{-1}$, and about $15 \%$ have $v>1000 \mathrm{~km} \mathrm{~s}^{-1}$; also, $10 \%$ have velocities less than 100 $\mathrm{km} \mathrm{s}^{-1}$. For the single-component model, only a small fraction of stars have 3-D velocities greater than $1000 \mathrm{~km} \mathrm{~s}^{-1}$ or less than $100 \mathrm{~km} \mathrm{~s}^{-1}$. As is evident in Fig. 3, the two-component distribution cleanly differentiates fast from slow pulsars, with a deficit of objects at intermediate velocities $\left(300-400 \mathrm{~km} \mathrm{~s}^{-1}\right)$. We compare the one- and two-component model likelihoods using a Bayesian odds ratio, marginalizing the posterior probability over the one and three parameters, respectively, to determine global likelihoods for the models. We find that the twocomponent model is favored $\sim 10^{4}$ to one.

Our best one-component velocity model is similar to that of Lyne \& Lorimer (1994), but the true distribution is apparently poorly described by a single component. Instead, the two-component distribution preferred by our analysis produces a significantly larger fraction of high-velocity pulsars (and a larger mean velocity, $\sim 540 \mathrm{~km} \mathrm{~s}^{-1}$ ) than has been required by any previous study of the radio pulsar population. We believe this is a consequence of (1) the unbiased nature of our analysis, in contrast to previous efforts that have not accounted fully for observational selection effects, and (2) our inclusion of deep, high-latitude surveys such as the Parkes $0.4 \mathrm{GHz}$ survey of the southerm sky (Manchester et al. 1996); see Sec. 3.5 for a detailed discussion of these effects.

We have explored models that include a third, very low velocity $\left(\leq 50 \mathrm{~km} \mathrm{~s}^{-1}\right)$ component. We find that such models produce only marginally improved likelihoods, and acceptable models permit at most $5 \%$ of NSs in such a low-velocity subpopulation. In terms of a Bayesian odds ratio, the small improvements in the overall likelihood are insufficient to support the added complexity of models that include a third velocity component. This result is similar to the finding of Cordes \& Chernoff (1998) but is more significant because our detection model accounts for selection against pulsars deep in the dispersing and scattering medium of the Galactic disk, to which low-velocity pulsars would be confined.

As shown in Table 1, we have also evaluated models that assume $n=4.5$ and no torque decay. The maximum likelihood of such models is smaller than the maximum likelihood for $n=3$ by three orders of magnitude for the same number of parameters, implying that canonical dipole spin-down is preferred over this one alternative braking model. We note, however, that the luminosity parameters $\left(\alpha, \beta, L_{0}\right)$ do not change significantly, nor does the mean birth magnetic field strength $\left\langle\log B_{0}[\mathrm{G}]\right\rangle$. The impact of braking-law assumptions is treated in more detail in the following section.

\subsection{Effects of Model Assumptions}


The maximum likelihood (ML) parameter values shown in Table 1 are valid in the context of assumptions made in our model-i.e., the quoted uncertainties are purely statistical, reflecting the $68 \%$ credible region defined by the likelihood function. Two fundamental assumptions may be particularly relevant to the our birth velocity results: spin-down through magnetic dipole braking, with no field decay, and the standardcandle hypothesis of pulsar luminosities. We discuss the potential effects of these assumptions below.

\subsubsection{Dipole $(n=3)$ braking.}

Because our likelihood function matches multiple simulated and observed pulsar properties simultaneously, at least two sources of velocity information are available: (1) the distribution of present-day Galactic scale heights of radio pulsars together with some estimate of their ages, and (2) proper motion measurements. We find that the spatial distribution alone significantly constrains birth velocities: if we ignore proper motion information, the peak of the posterior probability occurs at $\sigma_{v_{1}}=80 \mathrm{~km} \mathrm{~s}^{-1}, \sigma_{v_{2}}=350 \mathrm{~km} \mathrm{~s}^{-1}$, and $w_{1}=0.40$, consistent with the outcome based on the full likelihood, but with a smaller average velocity. Measured proper motions contribute preferentially to the high-velocity tail of the distribution because some middle-aged or old pulsars at low Galactic $|z|$ can masquerade as low-velocity objects until a proper motion measurement reveals that they are moving rapidly - the Guitar Nebula pulsar is a relevant example (Cordes, Romani \& Lundgren 1993).

Because spin-down governs the timescale on which neutron stars leaving the Galactic disk remain active as radio pulsars, the choice of braking index must affect the ML velocity model. We may qualitatively assess the effects of different spin-down laws and field decay as follows. Braking index measurements for the Crab and two other young pulsars yield values $n<3$; if this holds also for older objects, spin-down proceeds more slowly than we have modeled and, for a given pulsar, a lower characteristic birth velocity would be needed to reach the same Galactic $|z|$-height. Field decay, however, works in the opposite sense: if pulsar luminosities decay with decreasing field strength, as seems likely, field decay shortens the time in which an active pulsar must travel from its birthplace to its presentday vertical distance from the Galactic disk, and larger velocities than those we have derived would be necessary. The formal ML result in the analysis of Cordes \& Chernoff (1998) for braking index and field-decay timescale $\tau_{B}$ suggests $n \simeq 2.5$ and $\tau_{B} \simeq 6 \mathrm{Myr}$, or (with similar likelihood) $n \simeq 4-5$ and no field decay. The values of $n$ and $\tau_{B}$ in the first case would tend to alter our result in competing directions, while the rapid spin-down implied in the second case further enhances the high-velocity fraction in the derived velocity distribution, as demonstrated by our best model with $n=4.5$ (the mean velocity increases from $540 \mathrm{~km} \mathrm{~s}^{-1}$ to $660 \mathrm{~km} \mathrm{~s}^{-1}$ ). Furthermore, the proper motion measurements that boost the high-velocity component are not directly affected by details of the chosen spin-down law. We believe, therefore, that our primary conclusion, namely that a large fraction of Galactic NS are born with high velocity, is robust.

\subsubsection{Pulsars as standard candles.}

A unique aspect of our simulation is the description of observed pulsar fluxes as an explicitly modeled consequence of viewing orientation across a well-defined radio beam with intrinsic luminosity that depends only on period and spin-down rate, with no intrinsic spread. Past practice has been to allow for a range of observed fluxes (for fixed distance) from an ad-hoc distribution intended to mimic the combined effects of beaming orientation and any intrinsic spread in luminosity. Our explicit beaming model is a physically well-motivated improvement on past practice, and the standard-candle (for given spin parameters) hypothesis is the simplest allowed by the data. We investigate the possible consequences of such a fundamental change in the treatment of pulsar luminosities as follows.

In the top panel of Fig. 2, we show the distribution of observed fluxes, for a Vela-like young pulsar, that result from our beam and best-fit luminosity model (thick line) and from the smeared pseudo-luminosity distribution of Prószy ński \& Przybycień (1984) as modified by Hartman et al. (1997; see their Eqs. 2 and 3) (thin solid line), where a beaming fraction of 3 has been assumed for the latter, i.e., two-thirds of the simulated pulsars are assumed to be beaming away from the line of sight. Several points of comparison between the two flux distributions are noteworthy. First, the explicit beam model produces observed fluxes spanning a wider range than does the Hartman et al. smearing function, a somewhat surprising result that depends, of course, on the chosen shape of the beam (the outer "conal" component falls off as a Gaussian in our model). Second, the proportion of undetectable stars differs between the two models by less than a factor of two, a reflection of their effective beaming fractions. The difference is primarily due to the low-flux tail of the beamed distribution, detectable between 10 and $200 \mathrm{mJy}$-if the beam skirts were to fall off more rapidly than a Gaussian, the detection efficiency in our simulation would decrease, resulting in a higher birthrate through Eq. 9 (see Sec. 4.5). Third, when convolved by the Gaussian representing measurement error (dotted curve), the shape of the beamed distribution begins to resemble that of the distribution adopted to represent an intrinsic luminosity spread. Finally, the two distributions are offset in their absolute flux scales by nearly an order of magnitude. Most of this offset is due to different scalings of the luminosity with spin parameters: our best-fit luminosity law scales as $E^{1 / 2}$, whereas Hartman et al. assume $\dot{E}^{1 / 3}$. If the latter dependence were adopted for our beamed model, the resulting flux distribution would shift to the left, approaching the smeared pseudo-luminosity distribution.

To determine whether our beaming model alone produces a flux distribution sufficiently wide to describe the data, we have evaluated the likelihood for population models in which the relevant flux depends not only on the purely geometrical spread due to viewing orientation, but also on an additional, intrinsic spread in luminosity for objects with a given $P$ and $\vec{P}$. We modeled the intrinsic luminosity by a log-normal distribution with mean set by the scale parameter $L_{0}$ and coefficients $\alpha$ and $\beta$ as in Eq. 2, and with width equal to $\epsilon$ times the mean, where we treated $\epsilon$ as a parameter to be inferred. We examined the likelihood function as a function of $\epsilon$ and $L_{0}$, holding the other parameters fixed. We found that the likelihood decreases monotonically for all values $0 \leq \epsilon \leq 1$. Although we have not carried out a thorough search of parameter space for a global maximum of the likelihood, we interpret this behavior as evidence that geometric orientation effects are sufficient, without need for "hidden" variables, to account for the full spread in observed fluxes manifested by the radio pulsar population.

To provide a degree of separation between our luminosity model and the results of the likelihood analysis, we may also simply drop flux information from the likelihood function, by 
ignoring $\mathcal{D}_{b}$ observables (Sec. 2.3) and maximizing only the $\mathcal{L}_{a}$ function, Eq. 7. Assumptions inherent in the luminosity model then enter into the simulation only through a given pulsar's detectability. We find that the velocity and luminosity parameter values at maximum likelihood, and the inferred birthrate, change little (with the exception, as noted in the previous section, of a decreased average velocity) when flux and proper motion are eliminated from the likelihood function.

We conclude that the impact of our luminosity and beam assumptions on birth velocity results is likely confined to the effects of any insufficiency in the empirically-derived radio beam geometry we use, and then only indirectly. Further exploration of the effects of different beam properties is warranted and is underway.

\subsection{Comparison of Velocity PDF with previous results}

Several attempts to constrain the birth velocities of NSs have been described in the literature. (See Cordes \& Chernoff 1998 for a discussion of the analyses of Hansen \& Phinney 1997 and Lorimer et al. 1997). As emphasized earlier, our method offers an accounting of selection effects not previously available, rigorous treatment of uncertainties in, e.g., proper motion and distance, and analysis of the joint distribution of observable pulsar characteristics. Here, we compare our result to those of two recent studies.

Cordes \& Chernoff (1998; hereafter CC98) examined the kinematics of 49 pulsars younger than $10 \mathrm{Myr}$ with measured proper motions. Through a multi-dimensional likelihood analysis, but purposely not accounting for selection effects, they arrived at a birth velocity distribution for these objects described by $\sigma_{v_{1}}=175_{-24}^{+19} \mathrm{~km} \mathrm{~s}^{-1}, \sigma_{v_{2}}=700_{-132}^{+300} \mathrm{~km} \mathrm{~s}^{-1}$, and $w_{1}=0.86$. This distribution, shown in Fig. 3, significantly under-represents the high-velocity population implied by our analysis. To understand this difference, we have investigated the selection effects inherent in the choice of the 49 pulsars analyzed by CC 98 : because measurement of proper motion typically requires a time baseline of many years, all of the CC98 pulsars were discovered in some of the earliest pulsar surveys, which were substantially less sensitive than present-day surveys and directed along the Galactic plane. As a result, the CC98 pulsars, in addition to being young, constitute a volume-limited sample. We find that, if pulsars conform to our best-fit luminosity law, the detection efficiency of low-latitude, volume-limited surveys drops steadily with increasing velocity, so that at 1000 $\mathrm{km} \mathrm{s}^{-1}$ it is roughly one-third of its low-velocity value. In contrast, recent deep high-latitude (e.g., Foster et al. 1995; Thorsett et al. 1993) and all-sky (e.g., Manchester et al. 1996) surveys are sensitive to much of the volume occupied by high-velocity pulsars (away from the disk where propagation effects limit the survey volume). Such surveys combat the velocity-dependent selection effects to which early pulsar catalogs were subject. In this regard, an indirect observational bias against high-velocity pulsars remains unaccounted for in our analysis: by construction, our likelihood function assumes that proper motion measurements are available for an unbiased, representative subset of the pulsar catalog. In reality, such measurements have been attempted predominantly for nearby, bright pulsars that were discovered in early low-latitude surveys. Future proper-motion measurements of pulsars discovered in high-latitude surveys will mitigate this bias and directly probe the high end of the pulsar velocity distribution.

Fryer, Burrows \& Benz (1998; hereafter FBB) simulate binary evolution including NS kicks and compare the relative numbers of the resulting evolutionary endstates, LMXBs, HMXBs, and isolated pulsars, with observation. The analysis of FBB complements our own because they separate binary from kick velocities, but they do not account for selection effects associated with pulsar detection. FBB conclude that some $30 \%$ of NS are born with essentially zero kick, with the remaining $70 \%$ being given large $\left(500-600 \mathrm{~km} \mathrm{~s}^{-1}\right)$ kicks in addition to any orbital velocity retained following binary disruption. Our inferred birth velocity distribution is not inconsistent with this result: the low-velocity $\left(\sigma_{v_{1}} \simeq 90 \mathrm{~km} \mathrm{~s}^{-1}\right)$ component could be produced by binary break-up. This interpretation leads to other difficulties, however, in that the birthrate of binary endstates would be much larger than is observed if a significant fraction of NS (such as our $w_{1}=40 \%$ ) received zero kick at birth (Dewey \& Cordes 1987). FBB require only that their simulation yield birthrates of binaries no smaller than are observed, without penalizing models that generate too many binaries. The necessity for small kicks in FBB's analysis is dictated by their adopted $1 \%$ minimum retention fraction for globular clusters. Because most cluster pulsars have different evolutionary histories than NSs in the Galactic disk (e.g., as a result of collisional interactions and the higher binary fraction within clusters), birth velocity distributions relevant to disk pulsars may not apply to clusters (see Sec. 4.2). We suggest that the zero-kick fraction derived by FBB, $30 \%$, is an upper bound, and that our $90 \mathrm{~km} \mathrm{~s}^{-1}$ component is not due solely to relict orbital velocities. This conclusion is supported by the work of De Donder \& Vanbeveren (1999), who model the effects of binary membership of NS progenitors on the ultimate velocity distribution of single NSs, for a variety of binary properties. They find that the distribution of asymmetry-induced kicks dominates the space velocity distribution, with binary effects contributing an unimportant excess at low velocities, for any distribution of kicks with an average velocity $\gtrsim 150 \mathrm{~km} \mathrm{~s}^{-1}$. If a significant zero-kick component exists (e.g., the distribution suggested by FBB), most binaries survive and few low-velocity single pulsars remain.

\section{DISCUSSION}

\subsection{Asymmetric Kick Mechanisms}

It is widely believed that the large velocities imparted to neutron stars at birth arise through a combination of orbital disruption and a "kick" reaction to an asymmetric supernova explosion. Observational evidence for a kick impulse is found in a variety of systems: misalignments between the spin and orbital angular momentum axes of the relativistic NS-NS binaries B 1913+16 and B1534+12 (Wex, Kalogera \& Kramer 2000; Arzoumanian, Taylor \& Wolszczan 1998) and the presentday spin-orbit configuration of the binary pulsar J0045-7319 (Kaspi et al. 1996). Also, direct measurement of the proper motions of non-binary radio pulsars yield, in extreme cases, velocities far greater than can be provided by orbital motion, $\gtrsim 1000 \mathrm{~km} \mathrm{~s}^{-1}$. The Guitar Nebula pulsar, e.g., has a projected (two-dimensional) velocity $\sim 1600 \mathrm{~km} \mathrm{~s}^{-1}$, oriented nearly parallel to the Galactic plane (Cordes et al. 1993). Finally, the survival of binary systems into late evolutionary stages, e.g., NSNS, NS-white dwarf, or NS-black hole binaries, depends on the magnitude, frequency of occurrence, and preferred orientation (if any) of asymmetric kicks. The rates of survival of such systems have been estimated, without quantitative knowledge of the NS kick velocity distribution, through population synthesis models including orbital evolution (e.g., Dewey \& Cordes 
1987)—such efforts have affirmed the need for a velocity impulse on timescales shorter than or comparable to the orbital motion.

The kick magnitudes required by observations impose stringent constraints on theoretical models. No single mechanism for producing kicks as large as $1500 \mathrm{~km} \mathrm{~s}^{-1}$ has been conclusively identified; see Lai, Chernoff \& Cordes (2001; hereafter LCC) for a recent review of proposed mechanisms. Other clues to the nature of kicks have been scarce-the timescale on which kicks act is not known, and no correlation has been firmly established between the magnitudes or preferred directions of kicks and any other pulsar property (Deshpande, Ramachandran \& Radhakrishnan 1999). A possible exception is suggested by recent Chandra results for the Vela and Crab pulsars that show near alignment of the projected proper motion vectors and spin axes for these two young objects (but see Wex et al. 2000 for a possible counter-example). LCC explore the implications of such a correlation on proposed kick mechanisms.

For prompt natal kicks, two models have been explored in some detail. Large-scale density asymmetries in a presupernova core, seeded perhaps by energetic convection in inner burning shells driving overstable g-mode oscillations, can produce large kicks as a result of non-isotropic shock propagation and breakout during the explosion (see Lai 1999 for a review). The role of rotational averaging of the momentum impulse, however, is unclear-it may be difficult to produce spin-aligned kicks in this model (LCC) -and the existence of global asymmetries has not been verified numerically, as computational limitations have restricted simulations so far to consideration of two-dimensional models. Asymmetric emission of neutrinos in the presence of a strong magnetic field (due ultimately to CP violation in the weak interaction) can also impart momentum to a proto-NS, but impulses larger than a few hundred $\mathrm{km} \mathrm{s}^{-1}$ require very large magnetic fields ( $10^{15-16}$ Gauss; Arras \& Lai 1999).

An alternative to large impulsive kicks at birth is the electromagnetic rocket effect suggested by Harrison \& Tademaru (1975), which would accelerate a pulsar with an off-center magnetic dipole gradually over its initial spin-down timescale. This mechanism encounters two difficulties. Gravitational radiation due to unstable r-mode oscillations (e.g., Anderson 1998) may considerably enhance spin-down of a newborn neutron star so that the rocket effect acts on a much shorter timescale, resulting in a lower final velocity. Also, sufficiently large velocities may not be achievable on timescales short compared to the orbital periods of the progenitor systems that produce close NS-NS binaries or systems like PSR J0045-7319.

The significant fraction of the birth velocity distribution lying above $500 \mathrm{~km} \mathrm{~s}^{-1}$ cannot have its origin in presupernova orbital motion, strong evidence for asymmetric kicks through a mechanism that must be able to produce velocities at least as high as $1500 \mathrm{~km} \mathrm{~s}^{-1}$. On the other hand, our results also suggest that up to $40 \%$ of newborn NSs experience only a small, or zero, kick. It seems likely, then, that two mechanisms are needed, or that the mechanism producing large kicks (e.g., global hydrodynamic asymmetry) acts only in a subset of objects, perhaps through a threshold in the explosion energy or the spin rate of the progenitor core. As discussed above, kicks are needed to avoid producing too-many binary systems containing NSs, suggesting that some mechanism (e.g., asymmetric neutrino emission) responsible for small kicks is active in nearly all instances of core collapse. A bimodal birth velocity distribution is, how. ever, difficult to understand as a combination of independent mechanisms that produce large and small kicks (or a large kick and an uncorrelated residual velocity from disruption of an orbit). The convolution of two such independent processes should result in a broad uni-modal birth velocity distribution. Our analysis demonstrates, however, a clear preference for a distribution that can be adequately described (relative to any singleGaussian distribution) as a combination of two Gaussian components with very different dispersions.

\subsection{Escape from the Gravitational Potentials of the Galaxy and Globular Clusters}

Leonard \& Tremaine (1990) derive a lower bound of $V_{e}=430$ $\mathrm{km} \mathrm{s}^{-1}$ for the escape velocity from the Galactic potential in the solar neighborhood; the value of $V_{e}$ is larger at smaller Galactic radii. Assuming a typical escape velocity of $500 \mathrm{~km} \mathrm{~s}^{-1}$, we find that $50 \%$ of pulsars will be born with sufficient velocity to escape from the Galaxy for our two-component velocity model, or nearly $40 \%$ for the one-component model. The larger fraction is comparable to that derived by Lyne \& Lorimer (1994; hereafter LL94) based on their one-component distribution. CC98 derived an unbound fraction of $25 \%$, but argued that this figure was likely underestimated by a factor of two due to selection against high-velocities in their pulsar sample.

Drukier (1996) estimates the fraction of newborn NSs retained by globular clusters as a function of kick velocity, for both single stars and binaries containing NSs. It is generally believed that clusters must retain at least $10 \%$ of the NS born within them, but $V_{e}$ for a globular cluster is just $\sim 30 \mathrm{~km} \mathrm{~s}^{-1}$. For the LL94 distribution Drukier finds that at most $4 \%$ of NSs are retained for the most massive clusters, with typical fractions much smaller. Comparison of our preferred birth velocity distribution with LL94 suggests that the latter significantly underrepresents the low-velocity population of NS (as also suggested, e.g., by Hartman et al. 1997): in our preferred model, the fraction of objects with birth velocity $\lesssim 30 \mathrm{~km} \mathrm{~s}^{-1}$ is an order of magnitude larger than that for the LL94 distribution. To the extent that our conclusions for pulsars born in the disk are applicable to NSs in globular clusters, the enhanced low-velocity population in our model relative to LL94 should significantly increase the cluster retention rate over Drukier's findings. If our low-velocity component includes a significant contribution from pre-disruption orbital speeds, i.e., in the limit of zero kick for $w_{1}=40 \%$ of the population, the retention fraction is increased further, perhaps to as large as several tens of percent.

\subsection{Pulsar-Supernova Remnant Associations}

The supernova blast that accompanies NS birth drives a fast shock wave in the external medium. Our results for the birth velocity distribution of NSs may be used to estimate the fraction of objects that lie within their host supernova remnants (SNR), an important criterion used to assess proposed NS-SNR associations. We assume an explosion energy of $10^{51} E_{51}$ ergs in a medium of hydrogen density $n_{0} \mathrm{~cm}^{-3}$. For Sedov-Taylor expansion (Shull \& Draine 1987), the remnant scale at time $10^{4} t_{4}$ yrs is $R_{s}=12.5\left(E_{51} / n_{0}\right)^{1 / 5} t_{4}^{2 / 5} \mathrm{pc}$. A NS with birth velocity $v_{3} 10^{3} \mathrm{~km} \mathrm{~s}^{-1}$ passes through the edge of the remnant at $t_{4}=1.45\left(E_{51} / n_{0}\right)^{1 / 3} v_{3}^{-5 / 3}$. The shock becomes radiative (Shull 1987) at later times, $t_{\mathrm{sh}} \simeq 1.91 E_{51}^{3 / 14} n_{0}^{-4 / 7} 10^{4} \mathrm{yr}$, when the size is $R_{\mathrm{sh}}=16.2 E_{51}^{2 / 7} n_{0}^{-3 / 7} \mathrm{pc}$. Thence, $R=R_{\mathrm{sh}}\left(t / t_{\mathrm{sh}}\right)^{2 / 7}$ until the 
remnant slows to about $20 \mathrm{~km} \mathrm{~s}^{-1}$ and merges with the interstellar medium. In this regime, $t_{4}=1.51 E_{51}^{11 / 35} n_{0}^{-13 / 35} v_{3}^{-7 / 5}$.

The CDF for birth velocities less than $v$ is equivalent to the CDF for NSs lying within the remnant at time $t$ when $v$ and $t$ are related as above for both the Sedov-Taylor and radiative regimes. We show these CDFs, modified by the effects of projection for a distant observer, in Fig. 4 for several combinations of explosion energy and ambient density, as a function of NS age for our preferred birth velocity distribution. We find that up to $10 \%$ of pulsars younger than $20 \mathrm{kyr}$ will appear to lie outside of their host remnant shells. Such a small fraction is consistent with the set of known and proposed pulsar-SNR associations (e.g., Gaensler \& Johnston 1995). Of course, to assess the plausibility of a particular proposed association, age, distance, and especially proper motion measurements must be made.

\subsection{LIGO Rates and Binary Inspiral Sources of GRBs}

Both the number and orbital characteristics of binaries that survive a supernova explosion depend sensitively on the distribution of kick magnitudes. A number of studies (e.g., Dewey \& Cordes 1987; Wijers, van Paradijs \& van den Heuvel 1992; Brandt \& Podsiadlowski 1995; Portegies Zwart \& Spreeuw 1996; Lipunov et al. 1997; Bloom, Sigurdsson \& Pols 1999) have shown that the birthrates of double neutron star (DNS) and low- and high-mass $\mathrm{X}$-ray binary systems decrease with increasing characteristic kick velocity. For the DNS binaries, the resulting orbital period and eccentricity also determine the rate at which gravitational radiation is emitted; high-eccentricity and short-period systems will typically merge within $10^{9} \mathrm{yr}$, so that the rate of merger events will be smaller than the birthrate of all DNS binaries. In their final moments, merging systems are expected to produce gravitational radiation detectable by LIGO.

Assumed quantities (such as the importance of the high end of the initial mass function or the supernova rates in nearby host galaxies) contribute some uncertainty to DNS birthrate estimates, but the greatest source of uncertainty has been the unknown kick velocity distribution, yielding rates spanning in some cases more than an order of magnitude (e.g., from 9 to $384 \mathrm{Myr}^{-1}$ per galaxy in the work of Portegeis Zwart \& Spreeuw 1996). For the highest kick velocities that they model (a Maxwellian distribution with 3-dimensional velocity dispersion of $450 \mathrm{~km} \mathrm{~s}^{-1}$, their Model C), Portegeis Zwart \& Yungelson (1998) find a DNS birthrate of $17 \mathrm{Myr}^{-1}$ and a merger rate over $10 \mathrm{Gyr}$ of $12 \mathrm{Myr}^{-1}$. They also find that NS-black hole mergers will occur at a rate of 1 per Myr. Similarly, Bloom, et al. (1999) find a DNS birthrate of $3 \mathrm{Myr}^{-1}$ for their simulations incorporating large kicks (drawn from a Maxwellian distribution with one-dimensional dispersion of $270 \mathrm{~km} \mathrm{~s}^{-1}$ ). The birth velocity distribution that we have derived should improve such estimates significantly. Belczyński \& Bulik (1999) provide an expression linking the rate of DNS mergers to the kick velocity in one or more Gaussian components based on their binary evolution simulation, from which we infer a merger rate of 30 $\mathrm{Myr}^{-1}$. A general feature of two-component kick velocity distributions is that the resulting merger rate will be dominated by the small-kick component, but systems that remain bound following larger kicks also contribute through their shorter merger times owing to their high orbital eccentricities (see, e.g., Table 1 of BPS). The inclusion of a significant fraction of large kicks into binary evolution simulations will likely bring their birth and merger rate predictions in line with empirical estimates based on the small sample of known DNS systems (e.g., Arzoumanian, Cordes \& Wasserman 1999; Kalogera et al. 2001).

Models linking the coalescence of compact binaries with (at least some) gamma-ray bursts (GRBs) have been developed (Pacyński 1986; Goodman 1986), and can account for many properties of GRBs, including their energetics, spatial distribution, and (depending on assumptions about beaming) event rate. In this context, the discussion of DNS merger rates above applies equally well to GRBs-our inferred birth velocity distribution supports published estimates of the merger rate that assume the largest kicks. Bloom et al. (1999) model the effects of momentum impulses on the systemic motion of a compact binary in the gravitational potential of its host galaxy to determine the expected range of offsets on the sky between a GRB and the host galaxy. They find that the details of the distribution of kicks imparted to NSs at birth do not significantly affect these offsets because large kicks tend to disrupt the binary. A system's survival therefore acts as a velocity filter so that, for an ensemble of systems, our larger average kicks will not necessarily increase the spatial offsets expected of coalescing binaries containing NSs.

Binary systems containing a stellar-mass black hole are also potential sources of gravitational radiation for the LIGO and LISA detectors, and may drive certain gamma-ray bursts. The systemic velocities of low-mass $\mathrm{X}$-ray binaries containing black hole candidates (BHC-LMXBs) appear to be smaller than those of NS-LMXBs (White \& van Paradijs 1996). Black holes that form promptly upon the progenitor star's collapse are not expected to undergo kicks, but some black holes may form after passing through a short lived NS (or proto-NS) phase, e.g., if accretion of fallback material pushes the compact object's mass over the Chandrasekhar limit (see, e.g., Brandt, Podsiadlowski \& Sigurdsson 1995; hereafter BPS). Such "delayed formation" black holes may receive asymmetric kicks just as neutron stars do, with smaller kinematic consequences because of the larger mass of black holes. One BHC-LMXB, X-ray Nova Sco 1994, does have an unusually high peculiar velocity, $\simeq 110 \mathrm{~km} \mathrm{~s}^{-1}$, as do a few high-mass X-ray binaries. As pointed out by BPS, formation scenarios of Nova Sco are simplified if one invokes an asymmetric kick at birth; moreover, elemental abundances in its companion support a supernova origin for the black hole (Israelian et al. 1999). Extension of our radio-pulsar birth velocity distribution to black-hole systems would of course be speculative, but the properties of Nova Sco suggest that kicks similar to those associated with NSs also occur as part of some (perhaps rare) black-hole formation mechanisms. Any such kick would have implications for the survival rate of binaries containing a delayed-formation black hole. It should be noted that black-hole velocity distributions inferred from objects in binary systems may be prone to selection bias against high-velocity BHCs, the formation of which may have disrupted the host binary.

\subsection{Populations of Isolated Pulsars}

Early expectations that large numbers of old NSs accreting from the interstellar medium would be detectable in the soft $\mathrm{X}$ ray band have not been borne out by observations (Neuhäuser \& Trümper 1999). The earliest estimates, however, were based on an assumed NS velocity distribution peaking at $\sim 40 \mathrm{~km} \mathrm{~s}^{-1}$ (Treves \& Colpi 1991). The much larger average velocities inferred by LL94 and CC 98 suggested a solution to this discrepancy, as the accretion rate is expected to scale with velocity 
as $v^{-3}$; magnetic field decay on timescales less than $10^{9}$ years could also explain why so few ISM-accreting NSs are seen (for a review, see Treves et al. 2000). As described above, however, incomplete accounting for selection effects (those arising from radio-wave propagation in the Galactic disk) likely leads to an underestimated low-velocity proportion in the analyses of LL94, CC98, and others, potentially undermining the conclusion that the deficit of old accreting NSs is due to higher-thanexpected characteristic velocities. Our analysis models the effects of signal propagation and survey instrumentation, providing a more robust census of the low-velocity population. We infer a larger low-velocity fraction than do LL94 and CC98, a result which, together with our limit on an additional very low velocity component ( $<5 \%$ with birth velocity $<50 \mathrm{~km} \mathrm{~s}^{-1}$ ), should firmly constrain theoretical expectations for the number of nearby isolated NSs accreting from the ISM. As pointed out by Neuhauser \& Trumper (1999), the number of isolated NS detections in the ROSAT All-Sky Survey is consistent with estimates made by Madau \& Blaes (1994) based on a low birthvelocity distribution modified by diffusive heating of the NSs in the Galaxy-the resulting distribution peaks near $90 \mathrm{~km} \mathrm{~s}^{-1}$, similar to the low-velocity component of our preferred birth velocity model.

The observational phenomena known as soft-gamma repeaters (SGRs) and anomalous X-ray pulsars (AXPs) are believed to be associated with neutron stars. SGRs and AXPs are similar in many ways: high ( $\sim 10^{14} \mathrm{G}$ "magnetar") canonical field strengths, slow rotation (periods in the range 6-12 s), and luminosities that are too large to be due to magnetic dipole braking alone; alternate sources of energy are field decay and accretion of either fallback material from the supernova explosion or ejecta in the vicinity of the NS (e.g., Chatterjee, Hernquist \& Narayan 2000; van Paradijs, Taam \& van den Heuvel 1995). In addition to their distinct high-energy emission characteristics, a possibly significant observational difference between the two types of objects is that most AXPs are found near the centers of supernova remnants (so that associations are quite certain), while SGRs are found far from SNR centers, making associations less likely and requiring large $\left(\sim 1000 \mathrm{~km} \mathrm{~s}^{-1}\right)$ NS velocities if the associations are to be viable (Gaensler et al. 2001). Our results suggest that such high velocities are not rare among radio pulsars, certainly, and possibly among all NSs. Although distinctions between AXPs and SGRs on purely kinematic grounds remain controversial, models that invoke episodic interaction (e.g., accretion or spin-down due to the propeller effect) with fall-back disks or clumpy ejecta will clearly be influenced by the assumed velocities of the young neutron stars.

SGRs and AXPs may represent as much as half of the Galactic NS population (Gotthelf \& Vasisht 1999), yet they are distinct from radio pulsars in their spin characteristics and energetics, with no apparent overlap between the two populations. Estimates of the NS birthrate based on radio pulsars, including our own, must therefore be revised upward to account for these objects.

Our derived birthrate applies to isolated, rotation-powered, radio emitting pulsars, although the radio beam need not be visible to Earth-bound observers. Requiring non-binarity for our data sample results in the exclusion of just four pulsars that otherwise meet all of our selection criteria, suggesting that our birthrate essentially accounts aiso for radio pulsars in longlived, non-interacting binary systems (selection effects that re- duce survey sensitivities for binaries are unimportant for longperiod pulsars). From analyses of the local pulsar population, Lyne et al. (1998) derive a Galactic pulsar birthrate of one every 60 to 330 yrs, while Hartman et al. (1997) find modeldependent rates that cluster around $(300 \mathrm{yr})^{-1}$. Our birthrate estimate is a factor 2.5 lower still. Much of the discrepancy between our value and that of Hartman et al. (1997), however, can be attributed to the different effective beaming fractions in our analyses, evident in Fig. 2 (Sec. 3.4), suggesting perhaps that the low end of the Lyne et al. (1998) range is more appropriate. As emphasized earlier, our inferred birthrate depends on the assumed beam model and spin-down law (with no field decay), and cutoffs in the distribution of magnetic field strengths at birth. Disagreement between birthrate determinations is also likely due to different assumed radial distributions of birthsites in the Galaxy (Sec. 2.1.1). It is noteworthy that the majority of young cataloged radio pulsars were discovered in highfrequency $(\nu \sim 1.4 \mathrm{GHz}$ ) surveys of the Galactic Plane, and typically not detected, because of propagation effects, by the $0.4 \mathrm{GHz}$ surveys we have simulated. A future extension of our simulation to include high-frequency surveys will more directly probe the population of young pulsars near their birthsites, providing valuable constraints on their spatial distribution.

A number of radio-quiet but apparently rotation-driven (i.e., distinct from SGRs and AXPs) young neutron stars have recently been discovered at $X$ - and $\gamma$-ray wavelengths, often associated with supernova remnants (e.g., see Brazier \& Johnston 1999 for a compilation); these are most naturally described as pulsars whose radio beams do not intercept our line of sight, and so such objects would also be included in our birthrate estimate. However, Brazier \& Johnston (1999) derive a NS birthrate $\sim(91 \mathrm{yr})^{-1}$ by counting such objects and known radio pulsars within a distance of $3.5 \mathrm{kpc}$ and less than $20 \mathrm{kyr}$ old, and assuming a Gaussian radial distribution in the Galaxy. Again, our radio pulsar birthrate is substantially lower, raising the possibility that some young neutron stars may be truly radio quiet, i.e., not emitting radio beams. Such a conclusion, which cannot be ruled out at present, would be strengthened if a significant discrepancy between the birthrates of radio pulsars and young, radio-quiet NSs persists in future analyses of the Galactic neutron star population.

\section{SUMMARY}

Together with adjustable models of the birth, evolution, and beaming characteristics of the Galactic radio pulsar population, we have simulated eight large-area surveys, and accounted for observational selection effects, to infer the birth properties (velocity, period, magnetic field strength) of pulsars and their present-day radio luminosities. Our results suggest that the scaling of radio luminosity with spin parameters $P$ and $\dot{P}$ is similar to the scaling of voltage drop across the magnetic polar cap in pulsar magnetosphere models, and that old pulsars convert most of their spin-down energy into radio emissions. For birth velocities, we find that two-component distributions are preferred over one-component distributions, and we infer maximum likelihood velocities for the two (three-dimensional) Gaussian components of $90 \mathrm{~km} \mathrm{~s}^{-1}$ and $500 \mathrm{~km} \mathrm{~s}^{-1}$, with the population roughly equally divided between the two components. This result strongly supports existing evidence that neutron stars are subject to "kick" impulses at birth, presumably through asymmetric supernova explosions, and that the kick mechanism must be able to produce velocities of at least 1000 
$\mathrm{km} \mathrm{s}^{-1}$. We find that half of all pulsars born near or outside the solar circle will likely escape the Galaxy's gravitational pull. The large kicks implied by our birth velocity distribution also have important consequences for the survival rates, and final orbital configurations, of binaries in which a neutron star is formed, as well as for the plausibility of proposed pulsarsupernova remnant associations.

The catalog data that we have used to constrain our models provide spin parameters, fluxes, and Galactic locations for the known pulsars, but little in the way of direct velocity measurements through proper motions and accurate distances. Very Long Baseline Interferometry techniques now being perfected (e.g., Fomalont et al. 1999, Chatterjee et al. 2001) will provide high-quality proper-motion and parallax measurements for pulsars within $\sim 1 \mathrm{kpc}$, a substantial improvement to the current dataset. Future statistical analyses similar to the one presented here therefore promise important gains in our understanding of the velocity distribution of neutron stars.

We thank J. Arons, R. Bandiera, D. Lai, T. Loredo, D. R. Lorimer, A. G. Lyne, E. S. Phinney, M. Ruderman, and I. Wasserman for many fruitful conversations. C. Dolan and $M$. Jarvis contributed to the early development of simulation software. This research was conducted using the resources of the Cornell Theory Center, which receives funding from Cornell University, New York State, federal agencies, and corporate partners. Part of this work was performed while ZA held a National Research Council Research Associateship Award at NASA-GSFC. Support from NASA and NSF grants NAG 52851, NAG 5-8356, AST 92-18075, AST 95-30397, and AST 98-19931, is gratefully acknowledged.

\section{REFERENCES}

Arons, J., \& Scharlemann, E. T. 1979, ApJ, 231, 854

Arras, P., \& Lai, D. 1999, ApJ, 519, 745

Arzoumanian, Z., Cordes, J. M., \& Wasserman, I. 1999, ApJ, 520, 696

Arzoumanian, Z., Taylor, J. H., \& Wolszczan, A. 1998, in Pulsar Timing, General Relativity, and the Intemal Structure of Neutron Stars, eds. Z. Arzoumanian, F. van der Hooft, E. P. J. van den Heuvel (KNAW:Amsterdam)

Bloom, J. S., Sigurdsson, S., \& Pols, O. R. 1999, MNRAS, 305, 763 (BPS)

Brandt, N., \& Podsiadlowski, P. 1995, MNRAS, 274, 461

Brandt, N., Podsiadlowski, P., \& Sigurdsson, S. 1995, MNRAS, 277, L35

Brazier K T S \& Johnston, S. 1999, MNRAS, 305, 671

Belczyński, K., \& Bulik, T. 1999, A\&A, 346, 91

Caldwell, J. A. R., \& Ostriker, J. P. 1981, ApJ, 251, 61

Camilo. F., Nice, D. J., \& Taylor, J. H. 1996, ApJ, 461, 812

Chatterjee, P. Hemquist, L. \& Narayan, R. 2000, ApJ, 534, 373

Chatterjee, S., Cordes, J. M., Lazio, T. J. W., Goss, W. M., Fomalont, E. B., \& Benson, J. M. 2001, ApJ, 550, 287

Chen, K. \& Ruderman, M. 1993, ApJ, 402, 264

Cordes, J. M., \& Chernoff, D. F. 1998, ApJ, 505, 315 (CC98)

Cordes, J. M., Romani, R. W., \& Lundgren, S. C. 1993, Nature, 362, 133

D'Amico, N., Stappers, B. W., Bailes, M., Martin, C. E., Bell, J. F., Lyne, A. G. \& Manchester, R. N. 1998, MNRAS, 297, 28

De Donder, E., \& Vanbeveren, D. 1999, New Astr., 3, 167

Deshpande, A. A., Ramachandran, R., \& Radhakrishnan, V. 1999, A\&A, 351, 195

Dewey, R. J., \& Cordes, J. M. 1987, ApJ, 321, 780

Dewey, R. J., Taylor, J. H., Weisberg. J. M., \& Stokes, G. H. 1985, ApJ, 29, L25

Drukier, G. A. 1996, MNRAS, 280, 489

Fomalont, E. B., Goss, W. M., Beasley, A. J., \& Chatterjee, S. 1999, AJ, 117, 3025

Foster, R. S., Cadwell, B. J., Wolszczan, A., \& Anderson, S. B. 1995, ApJ, 454, 826

Fryer, C., Burrows, A., \& Benz, W. 1998, ApJ, 496, 333 (FBB)

Gaensler, B. M., \& Johnston, S. 1995, MNRAS, 277, 1243

Gaensler, B. M., Slane, P. O., Gothelf, E. V., \& Vasisht, G. 2001, ApJ, 559, 963

Goldreich, P., \& Julian, W. H. 1969, ApJ, 157, 869

Goodman, J. 1986, ApJ, 308, L17

Gotthelf. E. V. \& Vasisht, G. 1999, in Pulsar Astronomy-2000 and Beyond, eds. M. Kramer, N. Wex, \& R. Wielebinski (ASP: San Francisco)

Gregory, P. C., \& Loredo, T. J. 1992, ApJ, 398, 146

Hansen, B. M. S., \& Phinney, E. S. 1997, MNRAS, 291, 561

Hartman, J. W., Bhattacharya, D., Wijers, R. \& Verbunt, F. 1997, A\&A, 322, 477

Hughes, A., \& Bailes, M. 1999, ApJ, 522, 504

Israelian G. Rebolo, R., Basri, G., Casares, J., \& Martin, E. L. 1999, Nature, 401,142

Kalogera, V., Narayan, R., Spergel, D. N., \& Taylor, J. H. 200I, ApJ, 556, 340
Kaspi, V. M., Bailes, M., Manchester, R. N., Stappers, B. W., \& Bell, J. F. 1996, Nature, 381, 584

Lai, D. 1999, in Stellar Astrophysics (Proceedings of the Pacific Rim Conference, Hong Kong 1999), ed. K. S. Cheng (Kluwer: Dordrecht)

Lai, D. Chernoff, D. F., \& Cordes, J. M. 2001, ApJ, 549, 1111 (LCC)

Leonard, P. J. T., \& Tremaine, S. 1990, ApJ, 353, 486

Lipunov, V. M., Postnov, K. A., \& Prokhorov, M. E. 1997, MNRAS, 288, 245

Lorimer, D. R., Bailes, M. \& Harrison, P. A. 1997, MNRAS, 289, 592

Lyne, A. G. \& Lorimer, D. R. 1994, Nature, 369, 127

Lyne, A. G., \& Manchester, R. N. 1988, MNRAS, 234, 477

Lyne, A. G., et al. 1998, MNRAS, 295, 743

Madau, P. \& Blaes, O. 1994, ApJ, 423, 748

Manchester, R. N., et al. 1996, MNRAS, 279, 1235

Manchester, R. N., Lyne, A. G., Taylor, J. H., Durdin, J. M., Large, M. I., \&

Little, A. G. 1978, MNRAS, 185,409

Narayan, R. \& Ostriker, J. P. 1990, ApJ, 352, 222

Neuhauser, R., \& Trimper, J. E. 1999, A\&A 343, 151

Nice, D. J., Fruchter, A. S., \& Taylor, J. H. 1995, ApJ, 449, 156

Paczyński, B. 1986, ApJ, 308, L43

Paczýrski, B. 1990, ApJ, 348, 485

Portegies Zwart, S. F., \& Spreeuw, H. N. 1996, A\&A, 312, 670

Portegies Zwart, S. F., \& Yungelson, L. R. 1998, A\&A, 332, 173

Prószyński, M., \& Przybycień, D. 1984, in Millisecond Pulsars, eds. S.

Reynolds \& D. Stinebring (NRAO: Green Bank), 151

Rankin, J. M. 1993, ApJ, 405, 285

Ruderman, M. A. \& Sutherland, P. G. 1975, ApJ, 196, 51

Shull, J M. 1987, in Interstellar Processes, eds. D. J. Hollenbach \& H. A. Thronson, Jr. (Boston: Reidel), 225

Shull, J. M. \& Draine, B. T. 1987, ibid, 283

Spnit, H. C. \& Phinney, E. S. 1998, Nature, 393, 139

Stokes, G. H., Taylor, J. H., Weisberg, J. M., \& Dewey, R. J. 1985, Nature, 317,787

Taylor, J. H., \& Cordes, J. M. 1993, ApJ, 411, 674

Taylor, J. H. Manchester, R. N. \& Lyne, A. G. 1993, ApJSS, 88, 529

Taylor, J. H., Manchester, R. N., Lyne, A. G., \& Camilo, F. 1995, Radio Pulsar Catalog, hetp: //pulsar.princeton edu

Thompson. D. J., et al. 1999, ApJ, 516, 297

Thorsett, S. E. Deich, W. T. S., Kulkarni, S. R., Navarro, J., \& Vasisht, G. 1993, ApJ, 416, 182

Treves, A., \& Colpi, M. 1991, A\&A, 241, 107

Treves, A. Turolla, R., Zane, S. \& Colpi, M. 2000, PASP, 112, 297

van Paradijs, J., Taam, R. E., \& van den Heuvel, E. P. J. 1995, A\&A, 299, LAI

Wex, N., Kalogera, V., \& Kramer, M. 2000, ApJ, 528, 401

White, N. E. \& van Paradijs, J. 1996, ApJ, 473, L25

Wijers, R. A. M. J., van Paradijs, J., \& van den Heuvel, E. P. J. 1992, A\&A, 261, 145

Young, M. D. Manchester. R. N., \& Johnston, S. 1999, Nature, 400, 848

Zhang, B., Harding, A., \& Muslimov, A. 2000, ApJ, 531, L135 


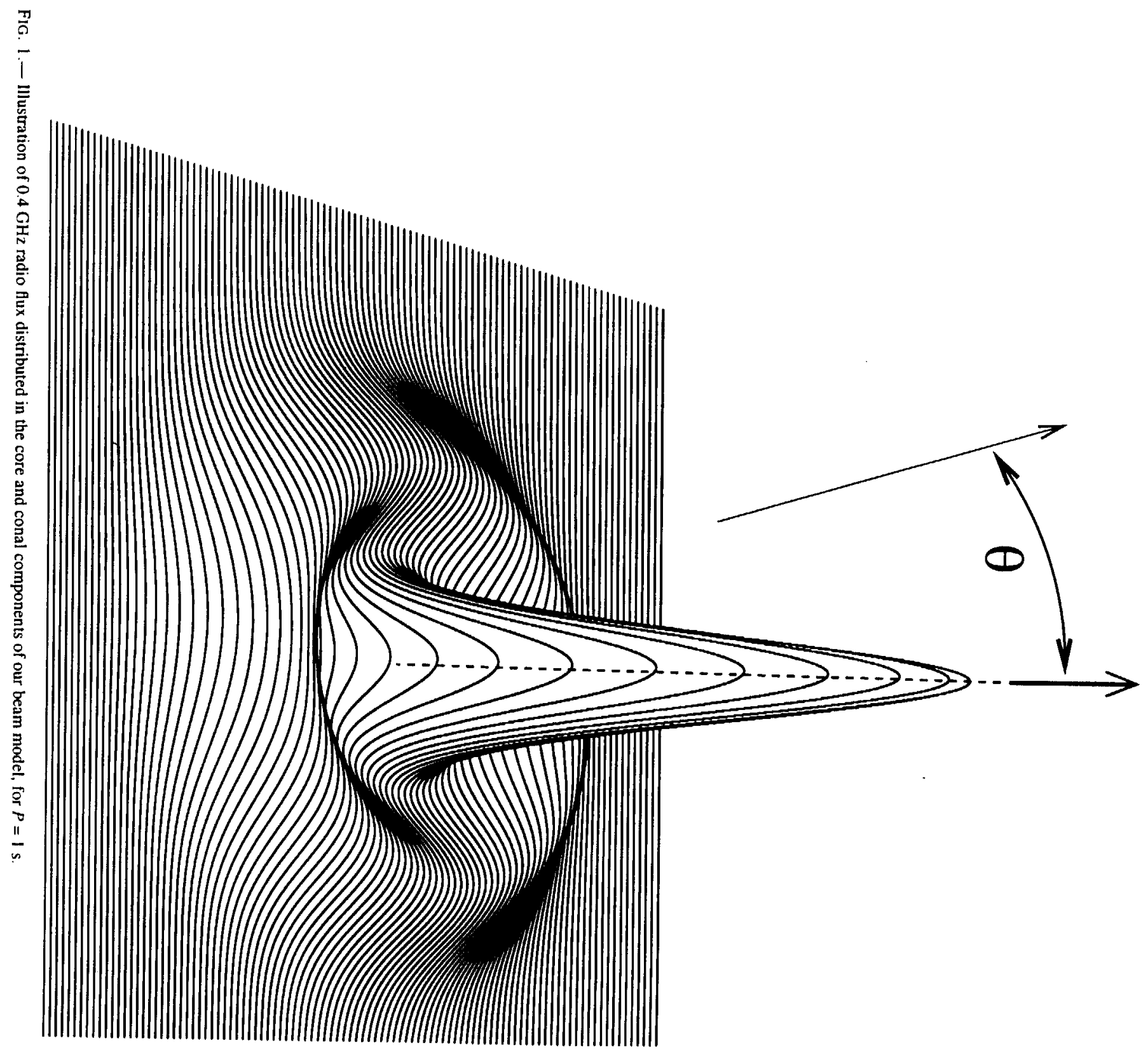




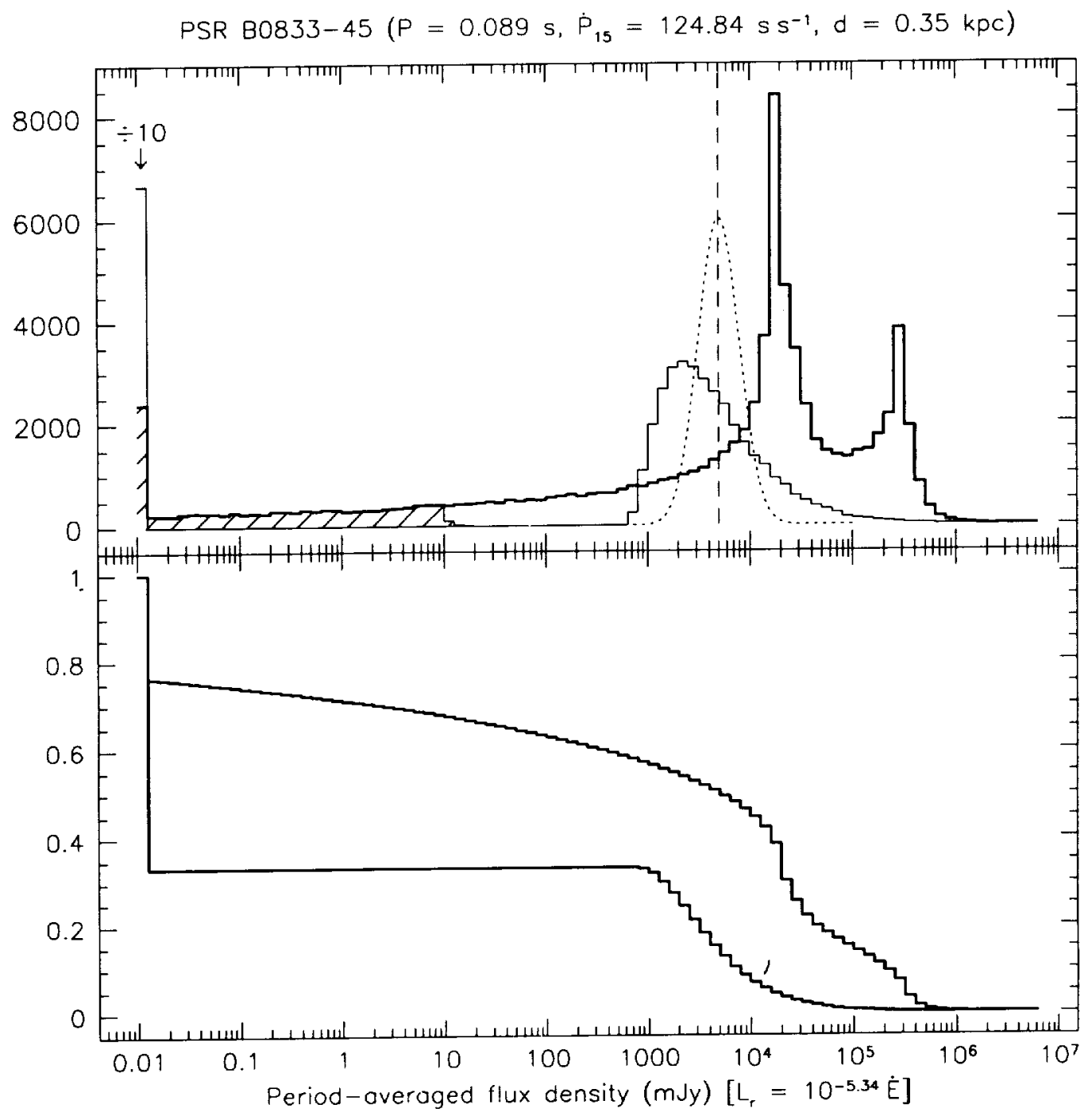

FIG. 2- The differential (top) and cumulative (bottom) distributions of period-averaged flux arising from: (thick line) our beam and best-fit luminosity models. eq. 12, given the period, spin-down rate, and distance of the Vela pulsar, for 10 randomly-drawn viewing geometries; (thin line) the spread in "pseudo-luminosity" eq. 12, given the period, spin-down rate, and distance of the dashed line indicates the pulsar's cataloged flux; the dotted Gaussian curve depicts the likelihood function used by Hartman et al. (1997), their Eqs. (2) and (3). The dashed line indicates the pulsar's cataloged lux, the dotted Gaussian curve depicts contion configurations that are undetectable we use to represent measurement error. See text, Sec. 3.4 . For our beam description, the hatched histo $0.4 \mathrm{GHz}$ survey (Manchester et al. 1996); the cumulative distribution indicates that a similar all-sky survey with a $10 \mathrm{mJy}$ flux limit will detect two-thirds of the Vela-like pulsars at the $350 \mathrm{pc}$ assumed distance. 


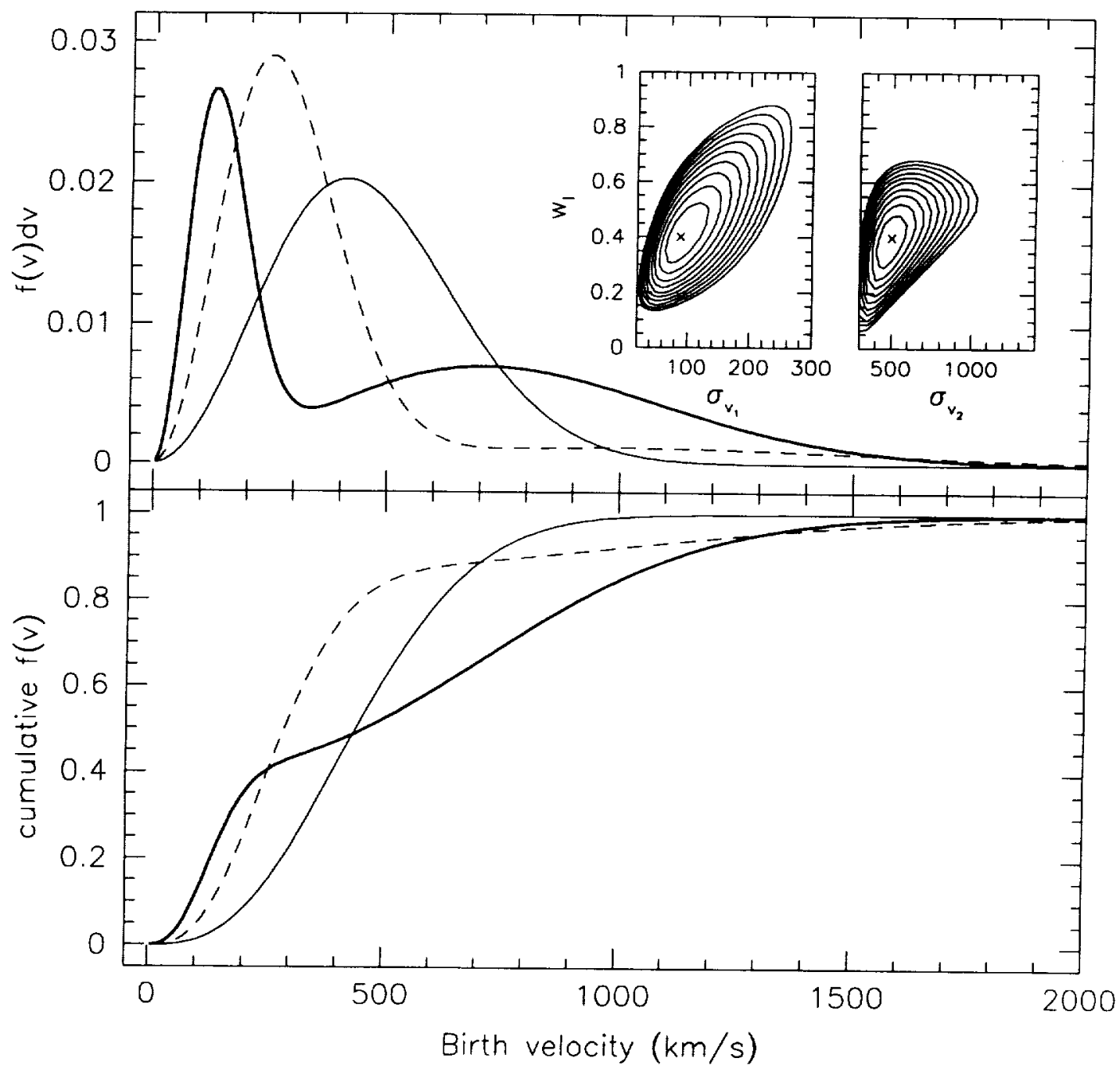

FIG. 3. - Differential and cumulative distributions of birth velocities of NSs. The heavy line represents the maximum-likelihood two-component distribution, with three-dimensional dispersions of the Gaussian components $\sigma_{v_{1}}=90 \mathrm{~km} \mathrm{~s}^{-1}$ and $\sigma_{v_{2}}=500 \mathrm{~km} \mathrm{~s}^{-1}$. with $w_{1}=40 \%$ of stars drawn from the low-velocity component. The thin line represents the best one-component Gaussian model, with dispersion $\alpha=290 \mathrm{~km} \mathrm{~s}^{-1}$. These results hold for magnetic dipole braking and no field decay. The dashed line represents the best-fit velocity distribution determined by Cordes \& Chernoff $\left(1998 ; a_{1}=175 \mathrm{~km} \mathrm{~s}^{-1}, \sigma_{v_{2}}=700 \mathrm{~km} \mathrm{~s}^{-1}\right.$, and $\left.w_{1}=86 \%\right)$ from a sample of young pulsars, without correcting for selection effects in pulsar surveys. Inset: Likelihood contours for the velocity parameters (all others held fixed at their maximum-likelihood values). The contour interval is $\Delta \ln \mathcal{L}=-2$ throughout. 


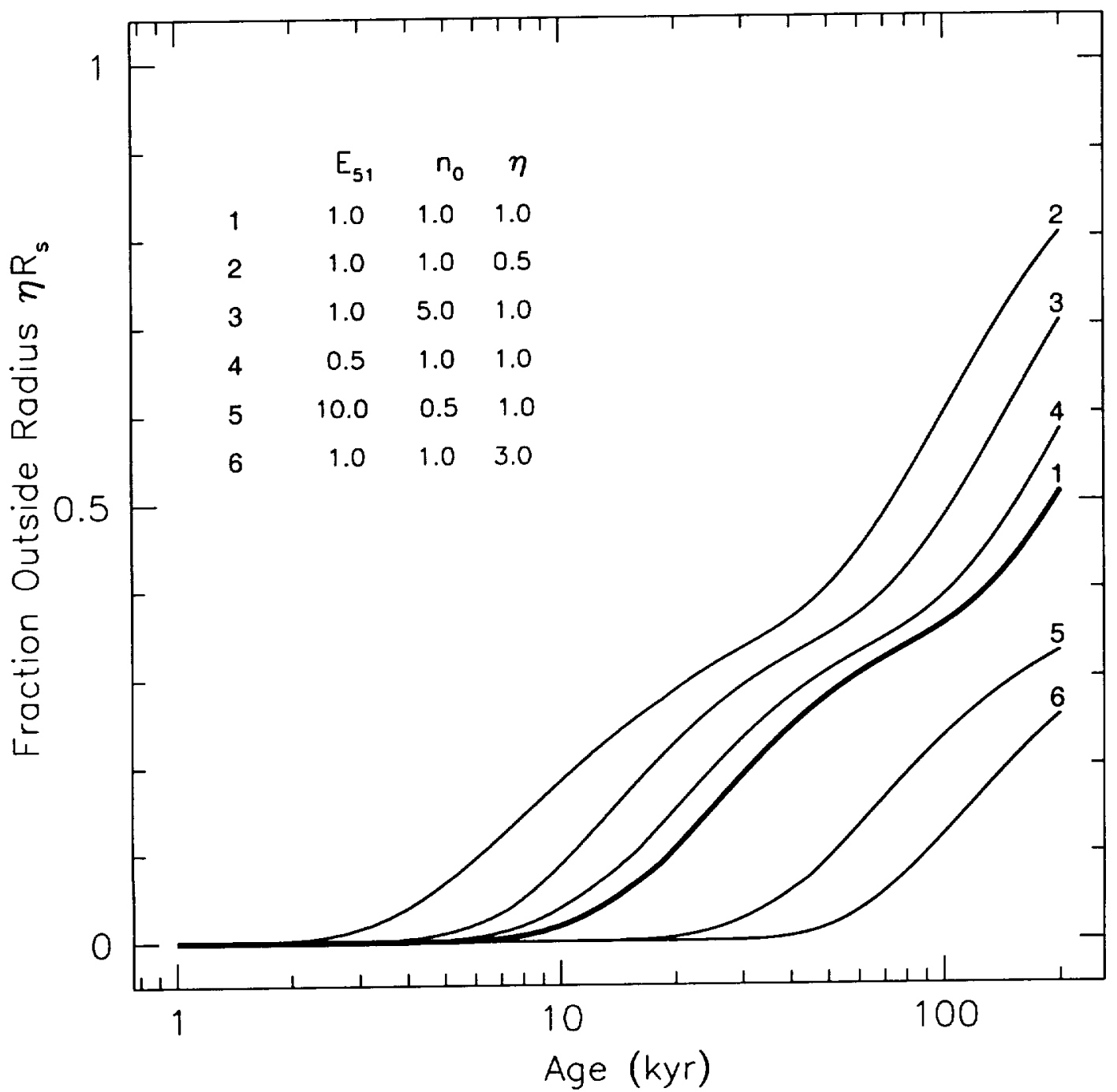

F1G 4-The fraction of neutron stars seen to be outside a characteristic radius of their host remnants (see text, Sec. 4.3). Some objects are physically outside F1G. 4.- The fraction of neutron stars seen to be outside a characteristic radius of their host remnants (see text, Sec. 4.3). Some cores different explosion energies,

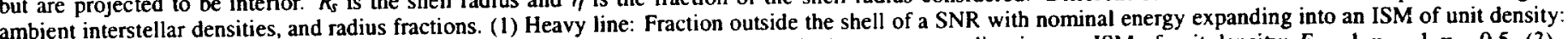
ambient interstellar densities, and radius fractions. (1) Heavy line: Fraction outside the shell of a SNR with inom an ISM of unit density: $\xi_{1}=1, n_{0}=1, \eta=0.5$. (3) $E_{51}=1, n_{0}=1, \eta=1$. (2) Fraction outside half the shell radius of a SNR with nominal energy expanding into an ISM of unit density: $5_{1}=1, n_{0}=1, \eta=0.5$. Fraction outside the shell of a SNR with nominal energy expanding into an ISM with larger density: $E_{1}=1, n_{0}=5, \eta=1$. (4) Fraction outside the shell of a SNR
with half nominal energy expanding into an ISM of unit density: $E_{51}=0.5, n_{0}=1, \eta=1$. (5) Fraction outside the shell of a hypernova with $\times 10$ nominal energy expanding into an underdense ISM: $E_{51}=10, n_{0}=0.5, \eta=1$. (6) Fraction outside three times the shell radius of a SNR with nominal energy expanding into an ISM of unit density: $E_{51}=1, n_{0}=1, \eta=3$. 
TABLE 1

SUMMARY OF MODEL PARAMETERS.

\begin{tabular}{|c|c|c|c|c|c|c|}
\hline \multirow[t]{2}{*}{ Parameter } & \multicolumn{3}{|c|}{ Peak posterior probability value $^{\mathrm{a}}$} & \multirow{2}{*}{$\begin{array}{c}\text { Credible } \\
\text { range }\end{array}$} & \multicolumn{2}{|c|}{ Range searched } \\
\hline & & & $n=4.5$ & & Min. & Max. \\
\hline$\alpha$ & \multicolumn{2}{|c|}{$\frac{n=3}{-1.3}$} & -1.6 & \pm 0.3 & -2 & 0.5 \\
\hline$\beta$ & \multicolumn{2}{|c|}{0.4} & 0.5 & \pm 0.1 & 0 & 1 \\
\hline $\log L_{0}$ & \multicolumn{2}{|c|}{29.3} & 29.1 & \pm 0.1 & 27.5 & 30.5 \\
\hline$\overline{\mathrm{DL}}$ & \multicolumn{2}{|c|}{0.5} & 0.8 & \pm 0.3 & -1.2 & 1.2 \\
\hline$\sigma_{\mathrm{DL}}$ & \multirow{2}{*}{\multicolumn{2}{|c|}{$\begin{array}{r}1.4 \\
-2.3\end{array}$}} & 1.4 & \pm 0.2 & 0 & 3.2 \\
\hline$\left\langle\log P_{0}[\mathrm{~s}]\right\rangle$ & & & -1.9 & \pm 0.2 & -2.6 & -1.6 \\
\hline$\sigma_{\log P_{0}}$ & \multicolumn{2}{|c|}{$>0.2$} & 0.6 & \pm 0.2 & 0.1 & 0.7 \\
\hline $\log P_{0}^{\text {lo }}$ (Note b) & \multicolumn{2}{|c|}{-4} & -4 & - & - & - \\
\hline $\log P_{0}^{\mathrm{hi}}$ & \multicolumn{2}{|c|}{0} & 0 & - & - & - \\
\hline$\left\langle\log B_{0}[\mathrm{G}]\right\rangle$ & \multicolumn{2}{|c|}{$\begin{array}{r}12.35 \\
0.40\end{array}$} & 12.35 & \pm 0.10 & 12.00 & 12.50 \\
\hline$\sigma_{\log B_{0}}$ & \multirow{2}{*}{\multicolumn{2}{|c|}{0.40}} & 0.65 & \pm 0.05 & 0.25 & 0.50 \\
\hline $\log B_{0}^{\mathrm{lo}}$ & & 11.2 & 11.2 & - & - & - \\
\hline $\log B_{0}^{\mathrm{hi}}$ & \multicolumn{2}{|c|}{13.8} & 13.8 & - & - & - \\
\hline$z_{0}(\mathrm{pc})$ & \multicolumn{2}{|c|}{160} & 220 & \pm 40 & 80 & 250 \\
\hline Velocity components $^{c}$ & One & Two & Two & & & \\
\hline$\sigma_{v_{1}}\left(\mathrm{~km} \mathrm{~s}^{-1}\right)$ & $290 \pm 30$ & $90_{-15}^{+20}$ & $120_{-15}^{+20}$ & & 50 & 400 \\
\hline$\sigma_{v_{2}}\left(\mathrm{~km} \mathrm{~s}^{-1}\right)$ & - & $500_{-150}^{+250}$ & $650_{-150}^{+250}$ & & 400 & 1400 \\
\hline$w_{1}$ & - & $0.4 \pm 0.2$ & $0.45 \pm 0.2$ & & 0 & 1 \\
\hline $\ln \left(\mathcal{L} / \mathcal{L}_{\max }\right)$ & -25.6 & 0 & -8.1 & & & \\
\hline
\end{tabular}

${ }^{a}$ The parameter value for the maximum likelihood (eq. 10) model, and with flat "priors" assumed.

${ }^{b}$ Lower and upper cutoffs of the $\log$-normal distributions for $B$ and $B_{0}$ were fixed at the values shown

'All trial distributions were truncated above $3000 \mathrm{~km} \mathrm{~s}^{-1}$. 